\title{
Does ethnic origin influence the incidence or severity of keratoconus?
}

\begin{abstract}
Purpose Keratoconus affects all races, yet very little information exists as to the relative frequency in patients of different ethnic origin. We aimed to establish the incidence and severity of keratoconus in Asian and white patients.

Methods The hospital records of the ophthalmology department of a large Midlands hospital with a catchment population of approximately 900000 (87\% white, $11 \%$ Asian, $2 \%$ other) were examined retrospectively for the 10 year period from 1989 to 1998.
\end{abstract}

Results For the age group 10-44 years the prevalence of keratoconus in Asians and whites was 229 and 57 per 100000 respectively, a relative prevalence of 4 to 1 . The incidence of keratoconus in the same age group was $\mathbf{1 9 . 6}$ and 4.5 per 100000 per year respectively, a relative incidence of 4.4 to 1 . Asians were significantly younger at presentation compared with whites (mean $22.3 \pm 6.5$ vs 26.5 \pm 8.5 years, $p<0.0001$ ). A first corneal graft was carried out on $14 \%$ of the Asian and $15 \%$ of the white patients. Of those having grafts, Asians were significantly younger than white patients at the time of diagnosis (mean $19.1 \pm$ 4.8 vs $25.7 \pm 7.3$ years, $p=0.005$ ) and at operation (mean $21.4 \pm 5.0$ vs $28.7 \pm 7.7$ years, $p=0.004)$. The interval from diagnosis to operation, though shorter for Asians, was not significantly different (mean $1.8 \pm 1.4$ vs $2.5 \pm$ 1.7 years, $p=0.2$ ).

Conclusion The results show previously unrecognised racial differences in the hospital presentation of keratoconus in the UK.

Compared with white patients, Asians have a fourfold increase in incidence, are younger at presentation and require corneal grafting at an earlier age.

Key words Ethnic origin, Incidence, Keratoconus

Keratoconus is believed to affect all races, yet very little information exists as to the relative frequency in patients of different ethnic origin. The incidence of keratoconus based on several studies has been estimated to lie anywhere from 2 to 600 cases per year per 100000 population. ${ }^{1}$ Much of this variation may reflect differences in the criteria used for diagnosis. However, it is possible that substantial differences may be due to the ethnic population studied. This may have implications in terms of understanding the aetiology of the disease and in resource planning, especially with regard to corneal grafting services. We aimed to establish the incidence and severity of keratoconus in Asian and white patients.

\section{Methods}

The hospital records of patients with keratoconus were examined retrospectively for the 10 year period from 1989 to 1998 . For diagnostic accuracy, analysis was restricted to patients referred for contact lenses or directly for corneal graft in whom a clinical diagnosis of keratoconus had been made by an ophthalmologist. The data recorded were the patient's name, sex, date of birth, address, religion, age at diagnosis, and age at corneal graft if performed. Patients living outside Leicestershire were excluded. The patient's name was used to identify those of Asian origin. This method has previously been shown to be highly reliable. ${ }^{2}$

The Leicester Royal Infirmary has a welldefined catchment population closely approximating the geographical area of Leicestershire, which has a population of almost 900 000. The 1991 Census data for this population were examined to establish the proportions of each ethnic group broken down both by sex and age groups (Table 1). The ethnic groups Indian, Pakistani, Bangladeshi and Other Asians were combined and termed Asian. Indians comprise $89.5 \%$ of this group.

The proportion of the population of ethnic origin other than white or Asian was only $2 \%$, and for the purposes of this study this proportion was considered to be negligible. The incidence of keratoconus in Asian and white patients was obtained by determining the number of new cases per year averaged over the 10 year period.
A.R. Pearson

N. Sarvananthan J.H. Sandford-Smith Department of Ophthalmology Leicester Royal Infirmary Leicester, UK

B. Soneji Department of Optometry Leicester Royal Infirmary Leicester, UK

Andrew Pearson Eye Administration Unit Leicester Royal Infirmary Leicester LE1 5WW, UK

Tel: $+44(0) 1162541414$

Fax: $+44(0) 1162585927$

Presented in part at the Oxford Ophthalmological Congress, 1999

Received: 28 October 1999 Accepted in revised form: 25 February 2000 
Table 1. Proportions of the population of each ethnic group in the age group 10-44 years for Leicestershire

\begin{tabular}{lcccccc}
\hline & \multicolumn{5}{c}{ Ethnic group (\%) } \\
\cline { 2 - 7 } & White & Asian & Black & Chinese & Other & Total \\
\hline Male & 87.1 & 10.9 & 1.1 & 0.3 & 0.6 & 100 \\
Female & 86.6 & 11.4 & 1.1 & 0.3 & 0.6 & 100 \\
Total & 86.8 & 11.2 & 1.1 & 0.3 & 0.6 & 100 \\
\hline
\end{tabular}

Figures are from the 1991 Census.

From all patients with keratoconus known to the content lens clinic, those who were still resident in Leicestershire were identified using the electoral register as of October 1998. Where the patient was not found on the register the patient's last known general practice or Leicestershire Health was contacted to establish whether the patient was still resident in Leicestershire. Prevalence was then estimated using the 1991 Census data.

\section{Results}

In total 382 patients with a diagnosis of keratoconus were seen for contact lenses or grafting. It was possible to establish whether the patient was still resident in Leicestershire in $100 \%$ of cases. There were 338 patients still resident as of October 1998. Of these, 112 (33\%) were Asian and 226 (67\%) were white. There were 271 new cases of keratoconus diagnosed between the years 1989 and 1998. Of these 271 patients, 96 (35\%) were Asian and 175 (65\%) were white.

For both Asians and whites keratoconus was more common in men than women (Asians $60 \%$ vs $40 \%$; whites $65 \%$ vs $35 \%$ ). Asians were significantly younger at presentation compared with whites (mean age $22.3 \pm 6.5$ years vs $26.5 \pm 8.5$ years, $t$-test $p<0.0001$ ). There was no significant difference in the age at diagnosis of men and women for Asians (21.8 vs 23.2 years, $t$-test $p=0.3$ ) or whites ( 26.2 vs 27.0 years, $t$-test $p=0.6)$. The relative proportions of each religion amongst the Asian patients with keratoconus were Hindu 44\%, Muslim 31\% and Sikh $25 \%$. For the overall Asian population of Leicester the approximate proportions of each religion are Hindu 62\%, Muslim 20\% and Sikh 18\% (Leicestershire City Council figures for 1983).

Using the 1991 Census data for the catchment population the overall incidence of keratoconus for Asian and white patients presenting to hospital and requiring contact lenses or grafting was approximately 11.8 per 100000 ( 1 in 8500 ) per year ( $95 \%$ confidence interval (95\% CI): 4.9-18.7) for Asians compared with 2.2 per 100000 ( 1 in 45000 ) per year (95\% CI: 0.8-3.6) for whites, a relative incidence of 5.3 to 1 . However, all but 7 of the patients (6 white, 1 Asian) were diagnosed between the ages of 10 and 44 years. In this age group the incidence in Asians was 19.6 per 100000 (1 in 5100) per year (95\% CI: $7.9-31.3$ ) and in whites 4.5 per 100000 (1 in 22 400) per year (95\% CI: 1.7-7.3). The relative incidence for this age group was therefore approximately 4.4 to 1 (males $4.2: 1$, females $4.7: 1)$.
Of the 338 patients with keratoconus resident in Leicestershire in October 1998, 226 (67\%) were white and $112(33 \%)$ were Asian. The prevalence in Asians was therefore 138 per 100000 and in whites 29 per 100000 population, a relative prevalence of 4.8 to 1 . For the age group 10-44 years the prevalence was 229 per 100000 for Asians and 57 per 100000 for whites, a relative prevalence of 4.0 to 1 .

Over the 10 year period 1989-1998 a first corneal graft was carried out on $13(14 \%)$ of the Asian patients and 26 (15\%) of the white patients. Of the Asians, $62 \%$ were male and $38 \%$ female; but for the whites $77 \%$ were male and $23 \%$ female. For white patients, therefore, a higher proportion of males progressed to grafting than females ( $18 \%$ vs $10 \%$ ). Seven of the 13 Asian patients requiring grafts were Muslim.

Asians were significantly younger than white patients both at the time of diagnosis (mean $19.1 \pm 4.8$ vs $25.7 \pm$ 7.3 years, $t$-test $p=0.005$ ) and at operation (mean $21.4 \pm$ 5.0 vs $28.7 \pm 7.7$ years, $t$-test $p=0.004$ ). The interval from diagnosis to operation was shorter for Asians than whites but this difference did not reach statistical significance (mean $1.8 \pm 1.4$ vs $2.5 \pm 1.7$ years, $t$-test $p=0.2$ ). There was no significant difference between male and female patients in terms of age at diagnosis or age at first graft, but for white patients males had a significantly shorter interval between diagnosis and graft $(2.0 \pm 1.5$ vs $4.0 \pm$ 1.8 years, $t$-test $p=0.01$ ).

\section{Discussion}

Our results show previously unrecognised racial differences in the hospital presentation of keratoconus in the United Kingdom. Asians have an incidence and prevalence of keratoconus more than 4 times that of whites. Our overall incidence figure for whites of 2.2 cases per 100000 per year accords well with that of Ihalainen $^{3}$ who found 1.4 cases per 100000 per year in a Finnish population and that of Kennedy et al. ${ }^{4}$ who found 2 cases per 100000 per year on review of the Mayo Clinic records for Olstead County, Minnesota. Several other series have estimated the incidence with figures as high as 600 per 100000 per year. ${ }^{5}$ It is likely that such wide variation mainly reflects differences in diagnostic criteria, in particular whether keratoscopy or corneal topography is used. We found no previous studies that looked at the possibility that ethnic origin might also influence the incidence of keratoconus.

We found figures for prevalence from only three studies. Ihalainen's figure of 28.8 per 100000 is virtually identical to our own figure in whites of 28.7 per 100000 . Kennedy et l. $^{4}$ estimated the prevalence at 54.5 per 100000 and, in Japan, Tanabe et al. ${ }^{6}$ estimated the prevalence at only 8.8 per 100000 .

The risk of keratoconus, therefore, is considerably higher in Asians than whites. In addition, the condition appears to be more severe in Asians. We found that Asians develop the disease at an earlier age, require corneal grafting at an earlier age and possibly show increased speed of disease progression compared with 
whites. Although we did not find a higher rate of grafting in Asians compared with whites over the 10 years studied, Tuft et al. ${ }^{7}$ did find that both blacks and Asians tended to progress to corneal grafting more frequently and more quickly than whites when studied over a longer period. In blacks, but not in Asians, this effect was at least partially explained by more severe disease at presentation. Further support for more severe disease in Asians is provided by a study by Tay et al. ${ }^{8}$ who report 29 corneal grafts carried out in 32 patients with keratoconus in Singapore. Of these, over half were in Indians and approximately one-third were in Chinese patients. Indians make up only $8 \%$ of the Singapore population compared with $72 \%$ Chinese, suggesting a relative rate of grafting in Indian patients in the order of 13 times that of Chinese. In neither study was a comment made regarding the age at onset of the disease or age at grafting in the different ethnic groups.

It is unclear why keratoconus is more common in Asians than whites. Several structural and biochemical abnormalities are associated with keratoconus but the precise aetiology remains unknown. We found that reliable information on atopic disease or eye rubbing, and non-inflammatory connective tissue disease were not available. However, these are not known to be more common in Asians and their role in the pathogenesis of keratoconus, if any, is not understood. The role of genetics in keratoconus is becoming clearer and it is likely that the observed difference in incidence reflects genetic differences. Almost $90 \%$ of Asians in

Leicestershire are of Indian extraction. It is interesting, therefore, in looking for a sub-population particularly affected, that amongst the Asians with keratoconus the observed proportion of each religion showed a higher than expected proportion of Muslims compared with the proportion of each religion in the community. This is in keeping with anecdotal evidence that the incidence of keratoconus is very high in Middle Eastern countries (J.H.S. personal observation). We had no information on the rates of consanguinity amongst Asians or whites though this could be relevant.

We found keratoconus to be substantially more common in men than women, for both Asian and white patients. This is in keeping with large series published by Tuft et al..$^{7}$ and Tanabe et al. ${ }^{6}$ Although some early studies showed a preponderance of females, the evidence now clearly shows that keratoconus is more common in males than females by a ratio approaching 2 to 1 . Tuft et al. found that males were also significantly younger at diagnosis. For both Asian and white males our results were similar, but this difference did not reach statistical significance. Finally, we found that, for whites, males progressed to grafting more quickly than females. This is contrary to Tuft et al.'s examination of risk factors for progression to grafting in a much larger series and may represent a chance statistical event predisposed by the small number of white females having grafts.

This study has a number of limitations. First, the data only include patients seen in the ophthalmology department and referred for contact lenses or corneal grafting. This selection method was used to ensure diagnostic accuracy so that an accurate relative incidence of keratoconus could be determined. However, it inevitably excludes patients with keratoconus managed in the community, those in the earliest stages of the disease where the diagnosis remains uncertain, and those who for whatever reason decide not to have a trial of contact lenses. Thus the true incidence and prevalence will be higher, but we had no reason to believe that any of these factors applied preferentially to Asians or whites.

Second, our estimates of incidence and prevalence relied on data from the 1991 Census for information on the catchment population of the Leicester Royal Infirmary. It is possible that a small number of patients with keratoconus living close to the Leicestershire boundary were referred to other ophthalmology units and thus do not appear in our figures. Although most of these patients are likely to be white we do not feel that the numbers are likely to alter the results significantly. Patients referred to the Leicester Royal Infirmary who did not live in Leicestershire were, as stated, excluded from the study.

The study can also be criticised for considering all non-Asian patients as white when identifying ethnic origin by name. We feel confident that this method accurately identifies Asians, including Asian women who marry whites, as both first name and surname were used. It is possible that some of the patients considered white may in fact be, for example, black or Chinese. However, only $2 \%$ of the population was other than white or Asian and the incidence of keratoconus in these groups would therefore need to be exceedingly high to alter the results significantly. The minimal data available do not suggest this. Moreover, Chinese patients can often be identified by name but did not feature in our results. Black patients could not be recalled as being more than a minimal proportion of contact lens fittings for those with keratoconus. For these reasons we feel that considering the $2 \%$ of the population that is neither white nor Asian to be negligible is unlikely to have had any significant effect on the results.

Finally, a significant limitation of the study is that it only provides information for whites and Asians. Tanabe et al.'s study ${ }^{6}$ suggests a prevalence of keratoconus in Japan of less than one-third that seen in whites. Clearly ethnic origin profoundly affects the risk of keratoconus, though comparative data for all ethnic groups remain unavailable. We found that ethnic origin was not generally recorded in hospital records. However, given accurate hospital records and a well-defined catchment population, the incidence in other ethnic groups, for example blacks and Chinese, could be established in areas where these groups make up a significant proportion of the population.

The authors would like to thank Rebecca McLean, research assistant, Department of Ophthalmology, for her assistance in identifying patients still resident in Leicestershire. 


\section{References}

1. Rabinowitz YS. Keratoconus. Surv Ophthalmol 1998;42:297-319.

2. Nicoll A, Bassett K, Ulijaszek J. What's in a name? J Epidemiol Community Health 1986;40:364-8.

3. Ihalainen A. Clinical and epidemiological features of keratoconus: genetic and external factors in the pathogenesis of the disease. Acta Ophthalmol Suppl 1986;178:5-54.

4. Kennedy RH, Bourne WM, Dyer JA. A 48 year clinical and epidemiological study of keratoconus. Am J Ophthalmol 1986;101:267-73.
5. Hofstetter HW. A keratoscopic survey of 13395 eyes. Am J Optom Am Acad Optom 1959;36:3-11.

6. Tanabe U, Fujiki K, Ogawa A, Ueda S, Kanai A. Prevalence of keratoconus patients in Japan. Nippon Ganka Gakkai 1985;89:407-11.

7. Tuft SJ, Moodaley LC, Gregory WM, Davison CR, Buckley RJ. Prognostic factors for the progression of keratoconus. Ophthalmology 1994;101:439-47.

8. Tay KH, Chan WK. Penetrating keratoplasty for keratoconus. Ann Acad Med Singapore 1997;26:132-7. 